\title{
REPRESENTATION OF FOURIER INTEGRALS AS SUMS. III ${ }^{1}$
}

R. J. DUFFIN

1. Introduction. Given an arbitrary function $\phi(x)$ let functions $f(x)$ and $g(x)$ be defined by the sums:

$$
\begin{aligned}
& f(x)=\sum_{1}^{\infty} \frac{\alpha_{n}}{n} \phi\left(\frac{x}{n}\right) \\
& g(x)=\sum_{1}^{\infty} \frac{\alpha_{n}}{x} \phi\left(\frac{n}{x}\right) .
\end{aligned}
$$

In the previous notes I [4] and II [5] it was shown that $f$ and $g$ are a transform pair; that is,

$$
g(x)=\int_{0}^{\infty} k(x t) f(t) d t
$$

provided $\alpha_{n}=\sin (\pi n / 2)$ and $k(x)=\sin (\pi x / 2)$.

Weinberger [8] treated the case when the coefficients $\alpha_{n}$ form a periodic sequence; that is $\alpha_{n+q}=\alpha_{n}$ for some integer $q$. Making use of the character theory of Dirichlet $L$-functions, he found the conditions on the $\alpha_{n}$ so that (3) holds with the kernel $\left(2 / q^{1 / 2}\right) \cos (2 \pi x / q)$ or the kernel $\left(2 / q^{1 / 2}\right) \sin (2 \pi x / q)$. (These kernels give self-reciprocal transforms for any value of $q$.) Weinberger's proofs hold under essentially the same conditions on $\phi$ as given in I.

Boas [1] made use of Poisson's summation formula to obtain representation formulae of the type (1) and (2) together with certain generalizations. In this connection it is of interest to note that the following Poisson summation formula

$$
\sum_{1}^{\infty} \alpha_{n} f(n x)=\sum_{1}^{\infty} \alpha_{n} g(n / x) / x
$$

is formally implied by (1) and (2). In fact both sides of (4) reduce to $\sum \sum\left(\alpha_{m} \alpha_{n} / n\right) \phi(m x / n)$.

The series in (1), (2), and (4) may be regarded as Möbius transforms. It is of interest to apply the Möbius inversion transform. The canonical form of the Möbius transforms [7] are

Received by the editors July 5, 1956.

1 The preparation of this note was sponsored by the Office of Ordnance Research, U. S. Army, Contract DA-36-061-ORD-490. 


$$
\begin{aligned}
& v(x)=\sum_{1}^{\infty} \alpha_{n} u(n x), \\
& u(x)=\sum_{1}^{\infty} \beta_{n} v(n x) .
\end{aligned}
$$

The constant coefficients $\alpha_{n}$ and $\beta_{n}$ satisfy the algorithm of Möbius,

$$
\alpha_{1} \beta_{1}=1, \quad \sum_{n=k} \alpha_{n} \beta_{m}=0 \quad \text { for } k>1 .
$$

The series on the left in (4) is seen to be a Möbius transform of type (5), so formally applying the inversion transform (6) gives

$$
f(x)=\sum_{1}^{\infty} \frac{\beta_{m}}{m x} \sum_{1}^{\infty} \alpha_{n} g\left(\frac{n}{m x}\right) .
$$

In I this formula was termed a direct representation of a Fourier integral as a sum.

The direct representation (8) is justified by Theorem 2, to follow, provided $g(x)$ is absolutely integrable and certain summability methods are used. Theorem 3 justifies (8) for the case $f(x)$ is of limited total variation. These theorems were announced in [3]; the proofs given here are substantially the same as then presented. In the meantime independent and interesting analyses of (8) have been given by Wintner [9] and by Goldberg and Varga [6].

2. A special summation formula. For the purpose of this paper it is sufficient to assume that $\alpha_{n}$ is multiplicative $\left(\alpha_{n} \alpha_{m}=\alpha_{n m}\right)$, bounded $\left(\left|\alpha_{n}\right| \leq A\right)$, and $\alpha_{1}=1$. It then follows that the Möbius algorithm (7) is satisfied by $\beta_{n}=\mu_{n} \alpha_{n}$. Here $\mu_{n}$ is the Möbius symbol $\mu_{n}=1,-1$, or 0 .

Let the symbol $\delta_{n}^{i}$ be defined as $\delta_{n}^{i}=0$ if the integer $n$ contains a prime factor greater than the $i$ th prime and $\delta_{n}^{i}=1$ otherwise. A series $\sum_{1}^{\infty} c_{n}$ will be said to be $P$ (rime) summable if $\sum_{1}^{\infty} \delta_{n}^{i} c_{n}$ has a limit as $\sum_{i \rightarrow \infty}$.

Lemma 1. Let $u(x)$ be defined for $x>0$ and let $u(x) \in L_{1}(1, \infty)$. Then the series (5) converges a.e. to a function $v(x)$. The series (6) is $P$ summable a.e. to $u(x)$.

Proof. According to Lemma 4 of I the series (5) converges absolutely almost everywhere. Let $x$ denote a point of absolute convergence. To apply the sieve of Erastosthenes let

$$
\begin{gathered}
v_{1}(x)=v(x)-\alpha_{2} v(2 x), \quad v_{2}(x)=v_{1}(x)-\alpha_{3} v_{1}(3 x), \\
v_{3}(x)=v_{2}(x)-\alpha_{5} v_{2}(5 x), \text { etc. }
\end{gathered}
$$


Clearly

$$
v_{i}(x)=u(x)+\sum_{2}^{\infty} \alpha_{n}^{i} u(n x)
$$

where $\alpha_{n}^{i}=0$ if $n$ contains any of the first $i$ primes as a factor; otherwise $\alpha_{n}^{i}=\alpha_{n}$. Let $i \rightarrow \infty$ then $\alpha_{n}^{i} \rightarrow 0$ and since the series is absolutely convergent it follows that $v_{i}(x) \rightarrow u(x)$. On the other hand it is seen from the definition of $v_{i}(x)$ and $\beta_{n}$ that

$$
v_{i}(x)=\sum_{1}^{\infty} \delta_{n}^{i} \beta_{n} v(n x)
$$

This proves Lemma 1.

Theorem 1. Let $\left(1+x^{-1}\right) \phi(x) \in L_{1}(0, \infty)$ then the series (1) and (2) converge a.e. and

$$
\sum_{1}^{\infty} \frac{\beta_{n}}{n x} g\left(\frac{1}{n x}\right)=\sum_{1}^{\infty} \frac{\beta_{n}}{n} f\left(\frac{x}{n}\right)
$$

a.e.

These series are $P$ summable.

Proof. Write (2) in the form $g(1 / x) / x=\sum_{1}^{\infty} \alpha_{n} \phi(n x)$. Then it is seen that Lemma 1 applies with $u(x)=\phi(x)$ so the left side of (9) is $\phi(x)$ for almost all $x$. If $\Phi(x)=\phi(1 / x) / x$ it is clear that $\left(1+x^{-1}\right) \Phi(x)$ $\in L_{1}(0, \infty)$. Write (1) in the form $f(1 / x) / x=\sum_{1}^{\infty} \alpha_{n} \Phi(n x)$. Thus by Lemma $1 \sum_{1}^{\infty}\left(\beta_{n} / n x\right) f(1 / n x)=\Phi(x)$ a.e. This shows that a.e. the right side of (9) is $\phi(x)$ and the proof is complete.

In I, $\alpha_{n}$ was taken to be $\sin (\pi n / 2)$; this makes $\alpha_{n}$ multiplicative, bounded, and $\alpha_{1}=1$. Under the hypothesis $\left(1+x^{-1}\right) \phi(x) \in L_{1}(0, \infty)$ it was shown that $f$ and $g$ are transforms relative to the kernel $\sin (\pi x / 2)$ provided suitable summability methods are used to evaluate (3). Thus in this sense (9) is a summation formula like that of Poisson.

It is not to be supposed that (9) is generally valid for functions satisfying (3). A counterexample is furnished by taking $f(x)$ to be the characteristic function of a closed interval on the positive real axis. Then the left side of (9) is continuous, but the right side is discontinuous. It would be of interest to know if the special summation formula (9) can be modified so as to obtain general validity.

3. An $L_{1}$ inversion theory. Suppose that $g(x)$ is the transform of $f(x)$ according to $(3)$ and that $g(x) \in L_{1}(1, \infty)$. In addition suppose that Poisson's summation formula (4) holds. Then application of Lemma 1 proves that the direct representation formula (8) holds (a.e. with $P$ summability). 
Various conditions for the validity of Poisson's formula are to be found in the literature [1]. A difficulty is that these conditions are somewhat restrictive. The procedure to follow avoids this difficulty by replacing Poisson's formula (4) by a modification easier to justify. For the sake of clarity attention will now be confined to the case $\alpha_{n}=1$; this is not essential.

Let $f(x) \in L_{1}(-\infty, \infty)$ then Poisson's integral formula for the upper half-plane is

$$
f(x, y)=\frac{1}{\pi} \int_{-\infty}^{\infty} \frac{y}{y^{2}+(x-t)^{2}} f(t) d t, \quad y>0 .
$$

Then $f(x, y)$ may be termed the harmonic continuation of $f(x)$ because $f(x, y)$ is harmonic and $f(x, y) \rightarrow f(x)$ a.e. as $y \rightarrow 0$. If $f(x)$ is given only for $x>0$ the (even) harmonic continuation of $f(x)$ is obtained by letting $f(-x)=f(x)$.

Lemma 2. Let $f(x) \in L_{1}(-\infty, \infty)$ and let

$$
g(x)=\int_{-\infty}^{\infty} e^{2 \pi i x t} f(t) d t
$$

Then for $y>0$

$$
\sum_{-\infty}^{\infty} e^{-2 \pi y|n|} g(n)=\sum_{-\infty}^{\infty} f(n, y)
$$

where $f(x, y)$ is the harmonic continuation of $f(x)$. Both series converge absolutely.

Proof. It is not difficult to derive the following identity

$$
\sum_{-\infty}^{\infty} e^{-2 \pi(y|n|-i t n)}=\frac{1}{\pi} \sum_{-\infty}^{\infty} \frac{y}{y^{2}+(n-t)^{2}} .
$$

Clearly both sides converge absolutely and boundedly. Thus it is possible to interchange summation and integration after multiplying by $f(t)$ and integrating. This proves the lemma.

THEOREM 2. Let $f(x) \in L_{1}(0, \infty)$ and let $g(x)$ be its transform relative to the kernel $2 \cos 2 \pi x$. Then the harmonic continuation of $f(x)$ is given by

$$
f(x, y)=\sum_{1}^{\infty} \frac{\mu_{m}}{m x} \sum_{1}^{\infty} e^{-2 \pi y n / m x} g\left(\frac{n}{m x}\right)
$$

where $x>0$ and $y>0$ and the sum on $m$ is evaluated by $P$ summability. Moreover $f(x, y) \rightarrow f(x)$ a.e. as $y \rightarrow 0$. 
Proof. It may be supposed that $f$ and $g$ are even, so

$$
\sum_{1}^{\infty} f(n x, y)=\sum_{1}^{\infty} e^{-2 n \pi y / x} g\left(n x^{-1}\right) x^{-1}+b x^{-1}+a .
$$

Here $a=-f(0, y) / 2$ and $b=g(0) / 2$. The series on the left is absolutely convergent so the method of proof of Lemma 1 applies. Since $v_{1}(x)$ $=v(x)-v(2 x)$ it follows that $v_{1}(x)=0$ for $v(x)=a$. Again if $v(x)=b x^{-1}$ then $\lim v_{i}(x)=b \pi\left(1-p_{j}^{-1}\right)$ where the product is over all primes $p_{j}$; this product is known to vanish. These considerations show that the terms $a$ and $b x^{-1}$ do not contribute and may be omitted to give formula (13).

4. A bounded variation theory. The conditions to be assumed now are quite different than in Theorem 2 ; moreover, summability methods are not needed.

THEOREM 3. Let $f(x)$ be a normalized function of limited total variation on the interval $(0, \infty)$ and let $f$ vanish at $\infty$. Then for $x>0$

$$
\sum_{1}^{\infty} \frac{-\mu_{2 m}}{2 m x} \sum_{1}^{\infty}(-1)^{n} f\left(\frac{n}{2 m x}\right)=2 \int_{0}^{\infty} \cos 2 \pi x t f(t) d t .
$$

If the summation on $m$ is multiplied by $x$ it is uniformly convergent.

Proof. A function is said to be normalized if $f(x+)+f(x-)=2 f(x)$. Then the Riemann-Stieltjes integral may be employed to write

$$
2 \sum_{1}^{\infty}(-1)^{n} f(n)=-f(0+)-\int_{0+}^{1-} d f+\int_{1+}^{2-} d f-\cdots .
$$

Let $\rho(x)$ be a periodic function of period 2 such that $\rho(x)=1 / 2$ for $0<x<1, \rho(x)=-1 / 2$ for $1<x<2$, and $\rho(0)=\rho(1)=0$. Then

$$
\sum_{1}^{\infty}(-1)^{n} f(n)=a-\int_{0+}^{1-} \rho d f-\int_{1+}^{2-} \rho d f-\cdots .
$$

Here $a=-f(0+) / 2$. It is convenient to write the sum of these integrals as a single Lebesgue-Stieltjes integral. Thus

$$
\sum_{1}^{\infty}(-1)^{n} f(m / 2 n x)=a-\int_{0}^{\infty} \rho(2 n x t) d f(t) .
$$

LEMMA 3. $\sum_{1}^{\infty}\left(-\mu_{2 n} / n\right) \rho(n x)$ converges uniformly to $(2 / \pi) \sin \pi x$.

Proof. This is a rather direct consequence of a theory developed by Davenport [2]. Davenport was concerned with the series $r(x)$ 
$=\sum_{1}^{\infty}(\pi n)^{-1} \sin 2 \pi n x$. It is known that $r(x)=1 / 2-x$ for $0<x<1$, hence $\rho(x)=2 r(x / 2)-r(x)=\sum_{1}^{\infty}\left(2 \alpha_{n} / \pi n\right) \sin \pi n t$. Here $2 \alpha_{n}$ $=1-(-1)^{n}$ and so $\beta_{n}=-\mu_{2 n}$. This shows that the lemma is at least formally correct.

To complete the proof it is convenient to refer to pp. 319 and 320 of Davenport's paper and to adopt the terminology employed there. His Theorem 2 states that $\sum_{1}^{\infty}\left(\mu_{n} / n\right) r(n x)$ converges uniformly to $(1 / \pi) \sin 2 \pi x$. The key results he used in the proof are Theorem 1 and Lemmas 12,13, and 14. Analogous theorems and lemmas are now set up. First it is observed that Lemma 12 holds with $\beta_{n}$ replacing $\mu_{n}$ merely by replacing $q$ by $2 q$. The analogy of Theorem 1 with $\beta_{n}$ replacing $\mu_{n}$ is achieved by noting that

$$
4 \sum \beta_{n} e^{\pi i n x}=\sum \mu_{n}\left(-e^{\pi i n(x+1)}+2 e^{\pi i n x}-e^{\pi i n(x-1)}\right) .
$$

Lemma 13 is now proved in the same way, but one less step is necessary since $\rho(x)$, unlike $r(x)$, is constant at points of continuity. Lemma 14 and Theorem 2 are paraphrased, and the proof of the Lemma 3 is complete.

Now relation (15) is multiplied by $-\mu_{2 m} / 2 m x$ and summed. On the right, summation under the integral sign is permitted because of the uniform convergence resulting from Lemma 3 . It is known that $\sum_{1}^{\infty} \mu_{2 n} / n=0,[2]$. Thus the right side reduces to $-\int_{0}^{\infty}(\pi x)^{-1} \sin 2 \pi x t$ $d f(t)$ and an integration by parts yields (14).

\section{REFERENCES}

1. R. P. Boas, Jr., Sums representing Fourier transforms, Proc. Amer. Math. Soc. vol. 3 (1952) pp. 444-447.

2. H. Davenport, On some infinite series involving arithmetical functions, Quart. J. Math. vol. 8 (1937) pp. 8-13 and 313-320.

3. R. J. Duffin, Möbius transforms and Fourier transforms, Bull. Amer. Math. Soc. vol. 47 (1941) p. 383.

4. - Representation of Fourier integrals as sums. I, Bull. Amer. Math. Soc. vol. 51 (1945) pp. 447-455.

5. - Representation of Fourier integrals as sums. II, Proc. Amer. Math. Soc. vol. 1 (1950) pp. 250-255.

6. R. R. Goldberg and R. S. Varga, Moebius inversion of Fourier transforms, Duke Math. J. vol. 24 (1956) pp. 553-560.

7. Einar Hille, The inversion problem of Möbius, Duke Math. J. vol. 3 (1937) pp. 549-568.

8. H. F. Weinberger, Fourier transforms of Moebius series, Carnegie Institute of Technology Thesis, 1950.

9. Aurel Wintner, The sum formula of Euler-Maclaurin and the inversions of Fourier and Möbius, Amer. J. Math. vol. 69 (1947) pp. 685-708 (See Theorem XV).

CARNEGie Institute of TechNology 